\title{
Development and validation of an instrument to assess oral health literacy in Norwegian adult dental patients
}

\author{
LINDA STEIN¹, KJELL SVERRE PETTERSEN², MAUD BERGDAHL, JAN \\ BERGDAHL $^{1}$
}

${ }^{1}$ Department of Clinical Dentistry, Faculty of Health Sciences, UiT The Arctic University of Norway, Troms $\emptyset$, Norway

2Department of Health, Nutrition and Management, Oslo and Akershus University College of Applied Sciences, Lillestrøm, Norway

Running head: Development of the AHLID

Correspondence: Linda Stein, Department of Clinical Dentistry, Faculty of Health Sciences, UiT The Arctic University of Norway, N-9037 Troms $\varnothing$, Norway. Telephone: $+47-77649142$. Fax: +47-77649101. E-mail: linda.stein@uit.no

Tables: 4

Figures: 0 


\begin{abstract}
Objective: To develop and validate an interview instrument to assess oral health literacy in Norwegian adult dental patients. Materials and methods: The instrument, Adult Health Literacy Instrument for Dentistry (AHLID), was based on an OECD instrument used to assess general literacy in adults. 130 Norwegian adults (mean age 48 years; $57 \%$ women) participated. AHLID included a selection of oral health related printed texts that ranged from 1 to 5 with respect to difficulty. A questionnaire regarding socio-demographic variables and knowledge of risk factors for oral disease was used. DMFT, stimulated salivary flow rate and streptococcus mutans and lactobacillus in saliva was also examined. Results: The Cronbach's alpha values of AHLID were 0.98 for internal consistency reliability $(\mathrm{p}<0.01)$ and 0.81 for test-retest reliability $(\mathrm{p}<0.05)$. AHLID score 3 was most frequent while very few fulfilled the criteria for score 1 and 5. Linear multiple regression analysis showed that lactobacillus in saliva and knowledge of risk factors for periodontitis and caries were predictor variables of AHLID score. Conclusions: AHLID appears to be reliable and valid to assess oral health literacy in Norwegian adults, and suggests a method for country-specific health literacy instruments.
\end{abstract}

Key words: health literacy, health literacy instrument, instrument validation, oral health literacy 


\section{Introduction}

In the International Adult Literacy Survey (IALS), literacy was defined functionally as understanding and employing printed information in daily activities at home, at work, and in the community [1]. Poor literacy skills among adults are common worldwide, and large proportions of adults have limited literacy skills even in the most economically advanced countries. IALS reported that between one-quarter and three-quarters of adults from 22 countries failed to attain a literacy level considered to be the minimum of skill level required to cope with demands of modern society [1]. In recent years, different literacies has been recognized, emphasizing that literacy is both content and context specific [2-4]. Individuals with higher levels of general literacy may not be able to consistently apply their knowledge and skills in situations requiring specific content knowledge, as well as in unfamiliar contexts [5]. A growing awareness of content-specific literacy in different contexts has been seen, which most likely led to the need of defining health literacy as a distinct concept. Today there are several definitions of health literacy, but it seems to be no commonly accepted definition [6]. Numerous studies have reported the prevalence of low health literacy. In the United States, the National Assessment of Adult Literacy survey (NAAL) indicated that $43 \%$ of the adult population had limited health literacy skills [7]. In the European Union, the recent Health Literacy Survey (HLS-EU) reported that $47 \%$ of the respondents had limited health literacy, indicating that health literacy is a challenge also in Europe [8]. Systematic reviews have found that low health literacy is associated with poorer health related knowledge and comprehension $[9,10]$, increased hospitalization and use of emergency care, decreased health preventive behaviour, as well as different health related outcomes [9]. Even though medical research highlights the importance of health literacy for patients' health knowledge and positive health outcomes, health literacy has received little attention in dentistry until recent years. Oral health literacy has been defined as the degree to which individuals have the 
capacity to obtain, process, and understand basic oral health information and services needed to make appropriate health decisions [11]. Oral health literacy includes the ability to understand instructions on prescription drug bottles, appointment slips, medical education brochures, dental professional's directions and consent forms, and the ability to negotiate complex health-care systems. There is some interesting research regarding oral health literacy in pediatric dentistry. Two studies have found associations between caregivers' oral health literacy and children's oral health $[12,13]$. Another study reported oral health literacy as a significant modifier of the association between caregivers' perceptions of their young children's oral health status and child oral health related quality of life [14]. In vulnerable populations, there has been found a significant relationship between oral health literacy and oral health knowledge among patients who are pregnant for the first time [15], and that oral health literacy of adults is associated with self-reported oral health in an indigenous Australian population [16]. A study from US found that increased oral health literacy is associated with better self-reported oral health status, and that self-efficacy may mediate the effect of oral health literacy on oral health status [17]. Other recent publications have demonstrated an association between oral health literacy levels and failing to show for dental appointments [18], and that low health literacy is associated with more severe periodontal disease [19].

To date, several oral health literacy instruments have been published. The original Rapid Estimate of Adult Literacy in Dentistry is based on the Rapid Estimate of Adult literacy in Medicine (REALM) [20], and consists in two versions, REALD-30 [21] and REALD-99 [22]. Both are reading recognition oral health literacy screening instruments, consisting of 30 or 99 item scales of chosen words based on oral etiology, anatomy, prevention and treatment. A revision of the REALD-30 has been published called the Two-Stage Rapid Estimate of Adult Literacy in Dentistry (TS-REALD) [23]. The TS-REALD format contains a routing 
test, which serves to define the respondent's initial literacy level, and three stage-two tests, which measure the respondent's health literacy score. Recently, another version of REALM, the REALMD-20 was published as a brief screening instrument for clinical use in medicine and dentistry [24]. To set the health literacy level, patients are asked to read aloud 20 medical and dental words. The Oral health Literacy Assessment in Spanish (OHLA-S) [25] also emerged from REALD-30. In addition to the word-recognition section there is a comprehension section using the multiple-choice format. The REALD-99 is also modified and translated into Chinese, published as the HKREALD-30 [26]. The Test of Functional Literacy in Dentistry (TOFHLiD) [27] is an instrument consisting of two sections, reading comprehension and numeracy skills. Respondents are asked to read the passages and fill in missing words that have been selectively omitted from the sentences. A list of four words is available as multiple-choice for the omitted words. The numeracy section consists of questions related to instructions specific to pediatric dentistry. The Test of Functional Health literacy in Adults (TOFHLA) [28] served as a template for the development of TOFHLiD as well as another instrument, Oral Health Literacy Instrument for Adults (OHLI) [29]. The reading comprehension is a 38-item test with words omitted from two passages, one on dental caries, and one on periodontal disease. Four possible choices are offered for each omitted word as multiple-choice. The Hong Kong Oral Health Literacy Assessment Task for Paediatric Dentistry (HKOHLAT-P) [30,31] is a Chinese instrument. HKOHLAT-P consists of three parts. Part one is a knowledge test, where parents are required to match dental pictures and words. Part two is a numeracy test with questions related to written oral health related information including numbers. Part three is a comprehension test consisting of three oral health related passages with fill-in blanks with four possible choices. The Health Literacy in Dentistry scale (HeLD) [32] is a newly developed scale for measuring oral health literacy 
in Indigenous Australians. It consists of 29 oral health literacy related items graded on a 5point Likert scale.

We did not consider any of the described English, Chinese or Spanish instruments for measuring oral health literacy in dentistry suitable for Norwegian adults. Given the fact that literacy involves understanding of words, translation is difficult due to some obvious barriers. Languages may differ in subtle ways, and words or phrases that are meaningful in one language and may have no exact counterpart in another language. In addition, there are some difficulties concerning the content of the instruments. As an example, comprehension of Medicaid rights in the United States is neither relevant nor authentic to the population in many European countries as oral health insurance is rarely used. Another aspect is that multiple-choice format is utilized in most health literacy instruments, which is not common practice in Norwegian and several other European educational systems. Furthermore, it has been recognized that health literacy might not be fully captured by word recognition instruments, as they are not meant to determine comprehension [21]. Due to differences between countries in language, culture, and health care systems, the need of country-specific instruments is obvious. Therefore, the objective of the present study was to develop and validate an interview instrument to assess oral health literacy in Norwegian adult dental patients.

\section{Materials and methods}

\section{Instrument development}

The instrument, Adult Health Literacy Instrument for Dentistry (AHLID) was adapted from the instrument used to measure general literacy by Organization of Economic Co-operation and Development (OECD) [33]. The OECD literacy instrument is developed by a group of researchers from several countries, and has been utilized to assess literacy levels in adults in 
two large international literacy surveys, IALS [1] and Adult Literacy and Life skills survey (ALL), [34]. The measurement of literacy in these studies involved tasks based on reading printed texts followed by answering a question related to the content. The OECD literacy instrument consists of several texts distinguished into five different levels of difficulty, ranging from one to five. The levels are set on the basis of three variables; type of match, type of information requested, and plausibility of distracting information [33]. Type of match refers to task difficulty, which increase with the amount of information in the question for which participants have to search in the text. Difficulty also increases with the number of responses that participants are asked to provide. In addition, task difficulty is influenced by the degree to which participants have to make inferences to match the given information in a question to corresponding information in the text, and to identify the requested information. Type of information required refers to the kinds of information that participants have to identify to answer a question successfully. The more concrete the requested information, the easier the task. Abstract information is considered to be of a higher level of difficulty. Plausibility of distractors concern the extent to which information in the text share one or more features with the information requested in the question, but do not fully satisfy what is requested. Tasks are judged to be easiest when no distractor information is present in the text and more difficult as the number of distractors increase. If the distractors share more features with the correct response, and the distractors appear in close proximity to the correct response, the task is considered even more difficult.

While the OECD instrument consists of printed texts selected for inclusion based on a broad range of context and content, AHLID consists of printed oral health information texts frequently used for the benefit of adult dental patients to complement communication with dentist and dental hygienists. The AHLID was pilot tested before the project started. Ten adults read several texts and answered questions related to each texts. The pilot testing 
indicated that a more definite method for registering answers was required, which resulted in a more structured interview guide with concrete aspects the interviewer could check for fully right, partly right or wrong/no answers. The ten most suitable texts were selected after the pilot testing, two for each of the five different oral health literacy levels. These printed texts included brochures on snuff, dental erosion, gingivitis, dental caries, and endodontic treatment. In addition, information letters giving instructions on how to use an occlusion splint, which precautions to take after tooth extraction, and post-operative information was included, as well as a prescription for candida treatment, and a consent form for dental treatment under general anesthesia. The broad range of oral health related information texts aimed to prevent that no single group of patients was advantaged or disadvantaged in terms of content familiarity or exposure.

Inspired by the OECD literacy instrument, the AHLID oral health literacy levels were set on the basis of the variables type of match, type of information requested, and plausibility of distracting information [33]. Like the OECD texts, the AHLID texts and accompanying questions also ranged from one to five with respect to difficulty (Table I). In a structured interview utilizing an interview guide, participants were asked to read the texts one by one, followed by a question from each of the texts. As the AHLID measurement was not supposed to be a test of participants' memory, they were prompted to search for the right answer in the text. The AHLID interview guide included questions and answers for all ten texts. As the purpose of utilizing printed texts in is providing oral health information to the patients, the nature of the questions was decided on basis of what was considered important in each text. The tasks started with texts at oral health literacy level one, and continued chronological up to level five. At least one out of two answers in each level had to be fully correct before continuing to texts at the next oral health literacy level. At the end of the interview, an oral 
health literacy score ranging from one to five was obtained according to participants' ability to answer the questions related to the texts.

\section{Sample and survey procedure}

Subjects were recruited from the waiting list of patients seeking care for the first time at the University Dental Clinic, Troms $\emptyset$, Norway. To be included in the study, participants had to be older than 20 years, master the Norwegian language, and have no severe visual impairment. Written consent was obtained prior to the investigation, and the information was repeated orally to ensure that participants understood what their participation in the study involved. In total, 146 adults gave their informed consent, among them two were excluded as they did not fit the inclusion criteria, eight were not available for participation in the data collection period, and six did not complete the questionnaire, which resulted in a number of 130 patients participating. The investigation was conducted at the Public Dental Service Competence Centre of Northern Norway, Troms $\varnothing$, Norway.

A structured interview utilizing the AHLID tasks was performed in order to assess the participant's oral health literacy score. The interviews took place in a suitable room free from disturbing noises, as well as any kind of dental equipment. All interviews were conducted by the same researcher who was trained by one of the authors who is an experienced dentist and psychologist (JB). The duration of the interviews ranged from approximately 7 to 25 minutes. Oral health variables were collected immediately after the AHLID interview was completed. All clinical examinations were conducted by an experienced dental hygienist who was blind to study participants' AHLID score. A calibration with one of the authors who is an experienced dentist (MB) was conducted prior to the data collection. Dentition status was examined utilizing the World Health Organization criteria whereby the number of Decayed, Missing and Filled Teeth (DMFT) are accounted for [35]. Stimulated salivary flow rate and 
measures of streptococcus mutans and lactobacillus in saliva were examined utilizing the Dentocult ${ }^{\circledR}$ SM strip mutans and the Dentocult ${ }^{\circledR}$ LB (Orion Diagnostica, Finland). Bacteria samples were scored from 0 to 3 according to the manufacturer's instructions [36,37]. Lactobacillus and streptococcus mutans in saliva were used as biomarkers for oral health as these bacteria are associated with sugar intake, oral hygiene, and presence of dentition surfaces like fillings and cavities [38]. Finally, participants were asked to complete a questionnaire about their sex, age, years of education, and knowledge of risk factors for the most common oral diseases, dental caries and periodontal disease. The research project was conducted in accordance with the World Medical Association Declaration of Helsinki, and approved by the regional ethical committee before the recruitment of participants started.

\section{Reliability and validity procedures}

When testing internal consistency reliability of AHLID, the research assistant (RA) was present when the principal investigator (PI) interviewed 20 participants. While the PI was in charge of conducting the AHLID interview, handled all the conversations, as well as checking answers utilizing the interview guide, the RA also checked for fully right, partly right or wrong/no answers on each task on a separate identical interview guide. The PI and the RA were seated at distance to each other and were not able to get vision of each other's interview guides. After the interview was completed, the score of the PI was noted in the protocol as the AHLID score, while the score assessed by the RA was used solely in the internal consistency reliability analyses. For the test-retest reliability, ten participants were randomly asked to come back to the dental clinic one to two weeks after the day of investigation. The AHLID measurement was then repeated. The retest was performed by the RA, who was calibrated with the PI, but blind to the AHLID score from the measurement conducted by the PI at baseline investigation. To ensure blinding, individuals participating in the test-retest analysis 
were not a part of the internal consistency measurements. Content validity was aimed by exclusively including brochures, prescriptions and information letters utilized in dental clinics nationwide or in the county at the time of investigation.

\section{Statistical analyses}

Internal consistency reliability between oral health literacy level determined by the PI and the RA was calculated statistically by using Cronbach's alpha. Cronbach's alpha was also used to statistically determine test-retest reliability. Descriptive statistics were conducted on recorded demographic data. We wanted to test AHLID's predictive validity, and found it reasonable to use variables other studies have reported to be associated with health literacy scores as predictor variables. In a first step, bivariate correlation analysis was performed to investigate associations between AHLID scores and sociodemographic variables, oral health variables, and oral health knowledge variables. Pearson correlation was used as a measure of monotone association used on continuous variables and the dichotomous variable gender. Spearman's correlation was used on the dichotomous oral health knowledge variable due to the difference in number of cases in each category. In the next step, a linear multiple regression analysis was performed with AHLID scores and statistical significant correlated variables found in the bivariate correlation analysis. Gender, age, years of education, and DMFT were used as control variables. Level of significance was set at 0.05 . All statistical analyses were performed using IBM SPSS Statistics software for Windows (version 19.0, IBM SPSS Inc., Chicago IL, USA).

\section{Results}

Of the hundred and thirty adult patients who participated in the study, $57 \%$ were women. The mean age (48 years) was similar for men and women, ranging from 21 to 80 years. The lowest 
education level was seven years, corresponding elementary school while 19 years was the highest, corresponding PhD-level. The mean for years of education was $13(\mathrm{SD} \pm 3.34)$ and there was no difference between men and women. Description and distribution of clinical oral health variables and oral health knowledge variables are presented in Table II. The Cronbach's alpha values of AHLID were 0.98 for internal consistency reliability $(\mathrm{p}<0.01)$, and 0.81 for test-retest reliability $(\mathrm{p}<0.05)$. The AHLID testing showed that almost half of the sample scored on oral health literacy level 3, few participants scored on levels 1 and 5, while the rest was almost equally distributed on levels 2 and 4 (Table I). Bivariate correlation analyses showed a statistically significant negative correlation between AHLID score and lactobacillus in saliva, and a positive correlation between AHLID score and knowledge of bacteria as a risk factor for periodontitis as well as knowledge of frequent meals as a risk factor for caries (Table III). In a linear multiple regression analyses, lactobacillus in saliva, knowledge of bacteria as a risk factor for periodontitis, and knowledge of frequent meals as a risk factor for caries were found to be predictor variables of AHLID score, controlling for DMFT, gender, age and years of education (Table IV).

\section{Discussion}

The objective of the present study was to develop and validate an interview instrument (AHLID) to assess oral health literacy in Norwegian adult dental patients. Reliability test gave a Cronbach's alpha value of 0.98 for internal consistency reliability, which indicates that AHLID measured the same underlying attribute when different persons evaluated the health literacy scores. Cronbach's alpha value was 0.81 for test-retest reliability. Taken together, the high values of both internal consistency and test-retest analyses show that AHLID seems to be a reliable instrument as a Cronbach's alpha coefficient of 0.70 or higher is considered satisfactory [39]. Regarding content validity, all printed texts included in AHLID were 
authentic oral health information materials utilized throughout Norway at time of investigation. Therefore, we consider that the instrument has sufficient content validity. Oral health information such as prescriptions, post-operative information, as well as several brochures and information letters on a broad range of oral health diseases, conditions and procedures, were included aiming to represent a variety of oral health information. However, all printed texts utilized in AHLID were developed for Norwegians, and if the instrument will be utilized in other countries, printed materials from the country of interest must of course be used due to differences in language, culture and society. Even in Norway, the materials utilized in AHLID require constant evaluation as available printed oral health information change over time. For English speaking populations, it can be argued that it will be more convenient to continue to use published health literacy instruments that already have been utilized in dental populations instead of adapting AHLID to other countries. Nevertheless, it has been proposed that reading recognition instruments like the different versions of REALM, which several of the published oral health instruments are based on, makes it difficult to determine if a patient really knows the meaning of a word or rather is simply able to pronounce it without having any knowledge of its meaning [22,39]. Critics have also argued that including numeracy in functional health literacy instruments, which has been done by some of the oral health literacy instruments, makes it difficult to isolate the relative effect [40]. Most published instruments for assessing oral health literacy are different versions based on the health literacy instruments REALM and TOFHLA, even though none of them are considered gold standard. Without an existing gold standard, it is reasonable to develop new instruments: which not necessarily are based on the before mentioned instruments, especially with a focus on country-specific instruments.

We found that over one quarter of the participants scored on oral health literacy level 1 or 2, which indicate that these individuals had severe problems understanding the authentic 
printed oral health information utilized in AHLID. This finding is in accordance with IALS and ALL, who reported that one out of three Norwegian adults scored on literacy level 1 or 2, showing that these people struggled to understand different kinds of information from printed texts $[1,34]$. Both studies included Norwegian adults aged 16-65 years representative for this population. Among published health literacy instruments for dentistry, TS-REALD and OHLI assessed similar health literacy levels as AHLID, while other instruments had a mean score. In the testing of TS-REALD, $19 \%$ were found to have low oral health literacy [23], while the corresponding figure for OHLI was only $3 \%$ [29]. However, the authors of OHLI state that their study population were young and well educated adults who were frequent users of dental care, which may explain the low percentage of people with lower health literacy levels. A systematic review of 85 studies describing the prevalence of limited health literacy in the United States revealed that over one quarter of subjects had low health literacy [41], which is similar to our results, although the studies did not assess oral health literacy. A recent health literacy survey, HLS-EU [8], reported limited health literacy in 29 to $62 \%$ of the participants. Some 8000 adults representative for the general population in eight European countries participated. However, no Nordic countries were included in the survey [8]. Patient information leaflets are used to support oral health promotion, treatment choice and decision making in dental settings. However, the health literacy level of the patients will most likely have an impact on how they acquire this information. Our results indicate that printed oral health information utilized in Norway today may be too complex and difficult to understand for many adults. Similar to our results, researchers have found that health information in general is written on a level too high for the majority of the population in the US [42]. Therefore, it might be reasonable to consider a critical review of the existing printed oral health information in Norway. 
Our results showed a significant correlation between high AHLID score and knowledge of both caries and periodontitis risk factors. The significant bivariate correlations were confirmed in multiple regression analyses. In the initial testing of the oral health literacy instrument OHLI [29], significant associations between health literacy scores and health knowledge scores were reported. In the health literacy research, many studies have demonstrated a relationship between health knowledge and health literacy [8-10].

We also found a significant correlation between low AHLID score and high count of lactobacillus in saliva. Because high counts of lactobacillus in saliva reflect the consumption of simple carbohydrates by the host over time [43], we interpret our results that those with low oral health literacy may not maintain their oral health as good as those with high oral health literacy. However, individuals with high levels of disease in earlier stages of the life course may have experienced substantial tooth loss that is now reflected as reduced bacterial levels, so we controlled for DMFT in the multivariate analyses. The association was still significant. Research describing the impact of oral health literacy on clinical objectively measured oral health of adults is scarce. No studies in oral health literacy research have used salivary biomarkers as objective clinical oral health measure. A study from US found a significant relationship between oral health literacy and periodontal status utilizing the word recognition instrument REALD-30 [18]. A study from Japan [44] found association between oral health literacy and clinical oral health such as DMFT and CPI. However, a validation of their method of assessing oral health literacy levels in the study is missing in the published paper. Finding a relationship between AHLID score and lactobacillus in saliva in our study strengthens the assumption health literacy researchers have found; that health literacy and health outcomes are connected [9]. However, the paucity of information regarding the relationship between oral health literacy and clinical oral health outcomes makes it important for more research to be conducted on this important topic. 
Our results should be considered in light of the study's limitations. First, we recognise that we were unable to validate AHLID with other oral health literacy instruments because an appropriate comparison instrument was not available in Norwegian. Second, we tested the instrument on a convenience sample. It cannot be ruled out that the participants who volunteered for this study may represent a population more interested in oral health information and/or less affected by the dental clinic context than the normal population. Third, the inclusion criteria only allowed Norwegian-speakers self-evaluated to master the language to participate, which might have inhibited certain immigrants and some Sami people to participate. Since some individuals in these groups are likely to be affected by limited oral health literacy, it is reasonable to believe that we would have found lower AHLID scores if more people representing these groups were included. Forth, due to the design of AHLID, participants need at least one correct answer on each level before continuing to texts at the next level. However, it cannot be ruled out that some participants would have been able to answer correctly on higher levels. Finally, it is important to understand the limitation of AHLID with regard to the definition of oral health literacy. It measures individuals' ability to process and understand basic printed oral health information, however not their ability to obtain oral health information.

Despite the limitations, AHLID appears to be reliable and valid, and provides a method for identifying patients who have difficulty understanding the content of important printed oral health information utilized in dental clinical setting in Norway today. Until now, only English, Chinese and Spanish oral health literacy instruments have been available. The need of country-specific oral health literacy instruments is obvious, and we are convinced that AHLID can be utilized as a template to measure oral health literacy in other countries. Given the fact that this instrument includes printed health information materials, it is a possibility to include other kinds of health information than oral health to assess health literacy in other 
fields of health. In future research, AHLID should be used to investigate if patients' oral health literacy levels can be utilized to enhance communication with patients and patients' oral health. 


\section{Acknowledgements}

The authors are grateful to the staff at the Public Dental Service Competence Centre of Northern Norway, Troms $\varnothing$, Norway as well as the staff at the University Dental Clinic, Troms $\varnothing$, Norway. We sincerely thank dental hygienist Hilde Nyborg for being research assistant in the data collection period. 


\section{References}

[1] OECD and Statistics Canada. Literacy in the information age: final report of the International adult literacy survey. Paris: OECD Publishing; 2000.

[2] Coiro J. Exploring literacy on the Internet. Reading comprehension on the Internet: expanding our understanding of reading comprehension to encompass new literacies. Read Teach 2003;56:458-64.

[3] Kozup J, Hogarth JM. Financial literacy, public policy, and consumers' self-protection more questions, fewer answers. J Consum Aff 2008;42:127-36.

[4] Primack BA, Hobbs R. Association of various components of media literacy and adolescent smoking. Am J Health Behav 2009;33:192-201.

[5] Nutbeam D. Defining and measuring health literacy: what can we learn from literacy studies? Int J Public Health 2009;54:303-5.

[6] Berkman ND, Davis TC, McCormack L. Health literacy: what is it? Health Com 2010;15:9-19.

[7] Kutner M, Greenberg E, Baer J. The health literacy of America's adults: results from the 2003 National Assessment of Adult Literacy. Washington, DC: US Department of Education, National Center for Education Statistics; 2006.

[8] HLS-EU Consortium. Comparative report of health literacy in eight EU member states. The European Health Literacy Survey (HLS-EU). 2012. Accessed 11 June 2014 online at http://www.health-literacy.eu

[9] Berkman ND, Sheridan MD, Donahue MD, Halpern DJ, Crotty K. Low health literacy and health outcomes: an updated systematic review. Ann Int Med 2011;155:97-107.

[10] Easton P, Entwistle VA, Williams B. Health in the hidden population of people with low literacy. A systematic review of the literature. BMC Public Health 2010;10:459. 
[11] US Department of Health and Human Services. A national call to action to promote oral health. Rockville (MD): US Department of Health and Human Services, Public Health Service, Centers for Disease Control and Prevention, National Institutes of Health, National Institutes of Dental and Craniofacial Research; 2003.

[12] Miller E, Lee JY, DeWalt DA, Vann WF. Impact of caregiver literacy on children's oral health outcomes. Pediatrics 2010,126:107-14.

[13] Bridges SM, Parthasaranthy DS, Wong HM, Yiu CKY, Au TK, McGrath CPJ. The relationship between caregiver functional oral health literacy and child oral health status. Patient Educ Couns 2014;94:411-6.

[14] Divaris K, Lee JY, Baker AD, Vann Jr WF. Caregivers' oral health literacy and their young children's oral health-related quality-of-life. Acta Odontol Scand 2012;70:390-7. [15] Hom JM, Lee JY, Divaris K, Baker AD, Vann WF Jr. Oral health literacy and knowledge among patients who are pregnant for the first time. JADA 2012;143:972-80.

[16] Parker E, Jamieson L. Associations between indigenous Australian oral health literacy and self-reported oral health outcomes. BMC Oral Health 2010;10:3.

[17] Lee JY, Divaris K, Baker AD, Rozier RG, Vann WF Jr. The relationship of oral health literacy and self-efficacy with oral health status and dental neglect. Am J Public Health; 2012;102:923-2.

[18] Holtzman JS, Atchison KA, Gironda MW, Radbod R, Gornbein J. The association between oral health literacy and failed appointments in adults attending a university-based general dental clinic. Community Dent Oral Epidemiol [online] 2013; doi:10.1111/cdoe.12089.

[19] Wehmeyer MMH, Corvin CL, Gutmiller JM, Lee JY. The impact of oral health literacy on periodontal health status. J Public Health Dent;2014;74:80-7. 
[20] Davis TC, Long SW, Jackson RH et al. Rapid estimate of adult literacy in medicine: a shortened screening instrument. Fam Med 1993;25:391-5.

[21] Lee JY, Rozier RG, Lee S-YD, Bender D, Ruiz RE. Development of a word recognition instrument to test health literacy in dentistry: the REALD-30 - a brief communication. J Public Health Dent 2007;67:94-8.

[22] Richman JA, Lee JY, Rozier RG, Gong DA, Pahel BT, Vann WF Jr. Evaluation of a word recognition instrument to test health literacy in dentistry: the REALD-99. J Public Health Dent 2007;67:99-104.

[23] Stucky BD, Lee JY, Lee S-YD, Rozier RG. Development of the two-stage rapid estimate of adult literacy in dentistry. Community Dent Oral Epidemiol 2011:39:474-80.

[24] Gironda M, Der-Martirosian C, Messadi D, Holtzman J, Atchison K. A brief dental/medical health literacy screen (REALMD-20). J Public Health Dent 2013;75:50-5. [25] Lee J, Stucky B, Rozier G, Lee S-Y, Zeldin LP. Oral health literacy assessment: development of an oral health literacy instrument for Spanish speakers. J Public Health Dent $2013 ; 73: 1-8$.

[26] Wong HM, Bridges SM, Yiu CKY, McGrath CJP, Au TK, Parthasarathy DS. Development and validation of Hong Kong Rapid estimate of adult literacy in dentistry. $\mathbf{J}$ Investig Clin Dent 2012;3:118-27.

[27] Gong DA, Lee JY, Rozier RG, Pahel BT, Richman JA, Vann, WF Jr. Development and testing of the Test of Functional Health Literacy in Dentistry (TOFHLiD). J Public Health Dent 2007;67:105-12.

[28] Parker RM, Baker DW, Williams MV, Nurss JR. The test of functional health literacy in adults: a new instrument for measuring patients' literacy skills. J Gen Intern Med 1995;10:537-41. 
[29] Sabbahi DA, Lawrence HP, Limeback H, Rootman I. Development and evaluation of an oral health literacy instrument for adults. Community Dent Oral Epidemiol 2009;37:451-62. [30] Wong HM, Bridges SM, Yiu CKY, McGrath CPJ, Au TK, Parthasarathy DS. Validation of the Hong Kong Oral Health Literacy Assessment Task for Paediatric Dentistry (HKOHLAT-P). Int J Paediatr Dent 2013;23:366-75.

[31] Bridges SM, Parthasarathy DS, Au TKF, Wong HM, Yiu CKY, McGrath CP. Development of functional oral health literacy assessment instruments: Application of literacy and cognitive theories. J Public Health Dent 2014;74:110-19.

[32] Jones K, Parker E, Mills H, Brennan D, Jamieson LM. Development and psychometric validation of a Health Literacy in Dentistry scale (HeLD). Community Dent Health 2014;31:37-43.

[33] Kirsch I. The international Adult Literacy Survey (IALS): Understanding what was measured. RTS Research Report RR-01-25. Princeton, NJ: Educational Testing Service; 2001.

[34] Statistics Canada and OECD. Learning a living. First results of the Adult literacy and life skills survey. Paris: OECD Publishing; 2005.

[35] World Health Organization. WHO oral health country/area profile programme (CAPP). Geneva: World Health Organization; 2000.

[36] Jensen B, Bratthall D. A new method for the estimation of mutans streptococci in human saliva. J Dent Res 1989;68:468-71.

[37] Larmas M. A new dip-slide method for the counting of salivary lactobacilli. Proc Finn Dent Soc 1975;71:31-5.

[38] Van Houte J. Role of micro-organisms in caries etiology. J Dent Res 1994;73:672-81.

[39] Nunnaly JC, Bernstein IH. Psychometric Theory. New York: McGraw-Hill; 1994. 
[40] Schonlau M, Martin L, Haas A, Decrose KP, Rudd R. Patients' literacy skills: more than just reading ability. J Health Commun 2011;16:1046-54.

[41] Paasche-Orlow MK, Parker RM, Gazmararian JA, Nielsen-Bohlman LT, Rudd RR. The prevalence of limited health literacy. J Intern Med 2005;20:175-84.

[42] Nielsen-Bohlman L, Panzer A, Kindig DA. (Eds). Health Literacy: a prescription to end confusion. Institute of Medicine of the National Academies, Committee on Health Literacy, Bord on Neuroscience and Behavioral Health. Washington (DC): The National Academies Press; 2004.

[43] Nishikawara F, Katsumura S, Ando A, Tamaki Y, Nakamura Y, Sato K, Nomura Y, Hanada N. Correlation of cariogenic bacteria and dental caries in adults. J Oral Sci 2006;48:245-51.

[44] Ueno M, Takeuchi S, Oshiro A, Kawaguchi Y. Relationship between oral health literacy and oral health behaviors and clinical status in Japanese adults. J Dent Sci 2012;8:170-6. 
Table I. Description of literacy levels and distribution of AHLID scores ( $\mathrm{n}=130)$

Reading a short text to locate a single piece of information which is

Level 1 identical or synonymous to the information given in the question.

Reading and locating a single piece of information in a relatively short

Level 2 text with plausible, but incorrect distracting information, or to integrate two or more pieces of information from the text.

Reading and making matches that require low-level inferences.

Level 3 Distracting information is present in the text, but is not located near the correct information.

Reading and performing multiple-feature matches as well as to integrate

Level 4 information from complex or lengthy passages.

Reading and searching for information in dense text which contains a

Level 5 number of plausible distractors. Participants may have to perform high level inferences in order to provide a correct answer to the question. 
Table II. Description and distribution of clinical oral health variables and oral health knowledge variables

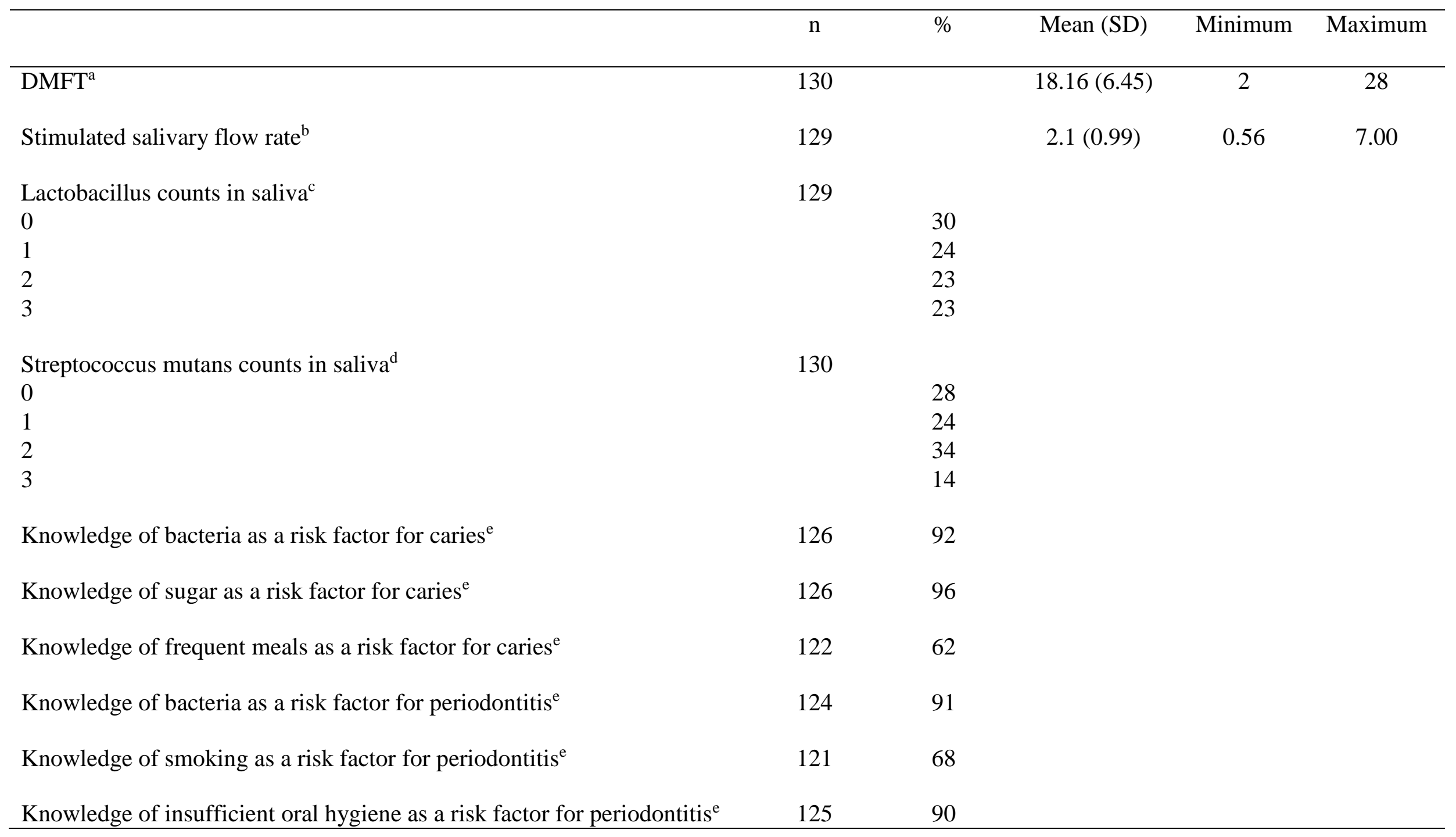


${ }^{a}$ Number of decayed, missed and filled teeth

${ }^{\mathrm{b}} \mathrm{ml} / \mathrm{min}$

${ }^{\mathrm{c}} 0=10^{3}, 1=10^{4}, 2=10^{5}, 3=10^{6}$

${ }^{\mathrm{d}} 0<10000,1=10000-100000,2=100000-1000000,4<1000000$

${ }^{\mathrm{e}}$ Correct response 
Table III. Bivariate correlation between AHLID scores and sociodemographic variables, clinical oral health variables, and oral health knowledge variables

\begin{tabular}{|c|c|c|c|}
\hline & $\mathrm{n}$ & $\mathrm{r}$ & p-value \\
\hline Gender $^{\mathrm{a}}$ & 130 & $0.084^{\mathrm{h}}$ & 0.341 \\
\hline $\mathrm{Age}^{\mathrm{b}}$ & 130 & $-0.071^{\mathrm{h}}$ & 0.425 \\
\hline Years of education & 130 & $0.070^{\mathrm{h}}$ & 0.429 \\
\hline DMFT $^{\mathrm{c}}$ & 130 & $-0.088^{\mathrm{h}}$ & 0.319 \\
\hline Stimulated salivary flow rate ${ }^{\mathrm{d}}$ & 130 & $0.157^{\mathrm{h}}$ & 0.076 \\
\hline Lactobacillus in saliva $^{\mathrm{e}}$ & 130 & $-0.209^{\mathrm{h}}$ & $0.018 *$ \\
\hline Streptococcus mutans in saliva ${ }^{\mathrm{f}}$ & 130 & $-0.003^{\mathrm{h}}$ & 0.937 \\
\hline Knowledge of bacteria as a risk factor for caries ${ }^{\mathrm{g}}$ & 126 & $0.129^{\mathrm{i}}$ & 0.151 \\
\hline Knowledge of sugar as a risk factor for caries ${ }^{\mathrm{g}}$ & 126 & $0.076^{\mathrm{i}}$ & 0.401 \\
\hline Knowledge of frequent meals as a risk factor for caries ${ }^{\mathrm{g}}$ & 122 & $0.305^{\mathrm{i}}$ & $0.001 * *$ \\
\hline Knowledge of bacteria as a risk factor for periodontitis ${ }^{\mathrm{g}}$ & 124 & $0.185^{\mathrm{i}}$ & $0.041 *$ \\
\hline Knowledge of smoking as a risk factor for periodontitis ${ }^{g}$ & 121 & $-0.039^{i}$ & 0.673 \\
\hline $\begin{array}{l}\text { Knowledge of insufficient oral hygiene as a risk factor } \\
\text { for periodontitis }^{\mathrm{g}}\end{array}$ & 125 & $0.011^{\mathrm{i}}$ & 0.904 \\
\hline $\begin{array}{l}{ }^{*} \mathrm{p}<0.05 \\
{ }^{* *} \mathrm{p}<0.01 \\
{ }^{\mathrm{a}} 0=\mathrm{man}, 1=\text { woman } \\
{ }^{\mathrm{b}} \text { Age in years } \\
{ }^{\mathrm{c}} \text { Number of decayed, missed and filled teeth } \\
{ }^{\mathrm{d}} \text { Measured as } \mathrm{ml} / \mathrm{min} \\
{ }^{\mathrm{e}} 0=10^{3}, 1=10^{4}, 2=10^{5}, 3=10^{6} \\
{ }^{\mathrm{f}} 0<10000,1=10000-100000,2=100000-1000000,4<10 \\
\mathrm{~g} 0=\text { incorrect response, } 1=\text { correct response } \\
\text { hPearson's } \mathrm{r} \\
{ }^{\mathrm{i}} \text { Spearman's rho }\end{array}$ & 0000 & & \\
\hline
\end{tabular}


Table IV. Linear multiple regression analyses on the AHLID with gender, age, years of education, DMFT, Lactobacillus, and oral health knowledge

\begin{tabular}{lccc}
\hline Predictor variables & $\mathrm{b}$ & $\mathrm{SE}$ & $\mathrm{p}$ \\
\hline Gender & -0.075 & 0.149 & 0.403 \\
Age & -0.058 & 0.008 & 0.660 \\
Years of education & -0.047 & 0.025 & 0.611 \\
DMFT & -0.001 & 0.017 & 0.996 \\
Lactobacillus in saliva & -0.218 & 0.064 & $0.016^{*}$ \\
Knowledge of bacteria as a risk factor for periodontitis & 0.218 & 0.280 & $0.023^{*}$ \\
Knowledge of frequent meals as a risk factor for caries & 0.320 & 0.152 & $0.001 * *$ \\
\hline $\begin{array}{l}* \mathrm{p}<0.05 \\
* * \mathrm{p}<0.01\end{array}$ & & & \\
\hline
\end{tabular}

